\title{
INTRODUCTION À L'CEUVRE DE BÉLAÏD AÏT ALI, comme première initiative d'écriture de tamazight en caractère latin
}

\author{
Mohand Ibrahim
}

\begin{abstract}
Résumé
Bélaïd Aït Ali était un écrivain, un conteur de langue kabyle. Il est célèbre pour avoir publié Les Cabiers en 1964, sous l'impulsion et le soutien des Pères Blancs dont le Père Degezelle. La rencontre ave le Père Degezelle eut lieu en 1945 quand celui-ci partait dispenser des soins aux habitants du village de Bélaid Aït Ali, Azrou. Père Degezelle propose à Bélaiid d'écrire quelques contes en langue kabyle et ce dernier écrit donc celui d'Aubépine.

Bélaïd prend goût à ce nouveau style d'écriture et découvre la profondeur et la délicatesse de la culture berbère. Il donne ainsi une âme à ses contes et transmet un intérêt au lecteur. En s'inspirant et en faisant mieux que ses prédécesseurs, Ben Sedira et Boulifa, il a donné une dimension nouvelle à la littérature kabyle en alphabet latin, notamment en dépassant les contraintes de loral. Il devient donc le pionnier de la littérature kabyle, tamazight, transcrite en alphabet latin.
\end{abstract}

Mots clés : Les Cahiers, Père Blancs, Père Degezelle, contes, kabyle, Aubépine, transcrire, alphabet, latin.

\section{Resumen}

Bélaïd Ait Ali era un escritor, un narrador en lengua cabileña. Es famoso por haber publicado Los Cuadernos en 1964, impulsado por los Padres Blancos, concretamente por el padre Degezelle. El encuentro con el padre Degezelle surgió en I945 cuando éste partió para atender a los habitantes del pueblo de Bélaid Ait Ali, Azrou. El padre Degezelle le propone a Bélaïd escribir algunos cuentos en lengua cabileña y éste escribe "Aubépine".

Bélaïd se enamora de este nuevo estilo de escritura y descubre la profundidad y la delicadeza de la cultura bereber. Sirve para dar un alma a sus historias y transmitir un interés al lector. Inspirándose y haciéndolo mejor que sus predecesores, Ben Sedira y Boulifa, dio una nueva dimensión a la literatura cabileña en alfabeto latino, especialmente sobrepasando las limitaciones de la oralidad. Por lo tanto, se convierte en pionero de la literatura cabileña, tamazight, transcrita en alfabeto latino.

Palabras claves: Los Cuadernos, Padres Blancos, Padre Degezelle, cuentos, cabileño, Aubépine, transcribir, alfabeto, latino. 
Fils d'une institutrice, Bélaïd a connu la langue française dès le berceau ${ }^{1}$. Son instruction s'est faite en français d'abord dans l'école de son village puis en France. Il n'a pas fait d'études universitaire à peine a-t-il fréquenté un collège. A I6 ans il se retrouve marié, avec une femme. Mais Bélaïd est doué d'une intelligence exceptionnelle. Non seulement il maîtrise à merveille la langue de "Molière ", mais il a une connaissance approfondie de son milieu kabyle. Fin observateur, curieux il emmagasinait lait tout ce qui est à sa portée. Grand rat des bibliothèques il assimilait tout ce qu'il lisait. Cette aptitude à comprendre son milieu a fait de lui ce grand écrivain en langue kabyle que nous connaissons. Il nous a laissé de belles pages écrites dans des moments difficiles de sa vie. Il a réussi à nous faire aimer les contes qu'il a rendus de vrais chefs d'œuvre littéraires. Il nous a décrits son village et les personnages qui habitaient avec une précision d'un artiste qu'il était. Car il fut aussi un peintre et un musicien. Il existe encore une aquarelle qu'il a offerte au Père Degezelle, son confident et son ami.

Bélaïd est né le 25 novembre 1909 à Bouira, ville où sa mère exerçait le métier d'institutrice. Bélaïd rentra dans son village d'Azrou Kollal à l'âge de 5 ans (vers I9I4) où il fut scolarisé jusqu'en I920. A cette date son demi frère Mohand Saïd le prit avec lui en France. Il y resta jusqu'en I925, année peut-être où il a perdu son père Ali At Ali. Il avait I6 ans et sa mère s'empressa de le marier à une jeune femme qui, d'après ses confidences était plus âgée que lui. Il la délaissa au bout de quelques mois

1 Mohand Ibrahim est natif du village d'Azrou Kollal, le même village que celui de Bélaïd Aït Ali dans le massif central de la Kabylie. Il fréquanta la même école qu'il quitta avec le certificat d'études primaires et intégra le monde du travail à un âge précoce. Autodidacte, assoiffé de culture, il ne cessa de poursuivre sa formation en suivant des cours du soir après son travail. Dans les années 1980 il retrouve presque par hasard le Père Degezelle qu'il a connu en Kabylie. Depuis cette rencontre, leur principale discussion portait sur Bélaïd. Le Père ne cesse de pousser son interlocuteur à faire quelque chose quand celui-ci lui demandait pourquoi « les cahiers » ne sont pas réédités. Il lui fournit des documents en particulier l'exemplaire du livre édité en 1964 avec les annotations du Père. Il ne reste plus à notre auteur à se mettre au travail. C'est ainsi qu'il s'inscrit à l'inalco pour parfaire ses connaissances sur berbère en général et le kabyle en particulier. C'est ainsi qu'il obtient un diplôme de maîtrise dont le sujet fut une recherche sur la vie et l'œuvre pionnière de Bélaïd Aït Ali dont il tira un ouvrage intitulé : Bélaïd Aït Ali. Errance ou génie littéraire. Mohand Ibrahim s'est attelé aussi à la réécriture moderne de l’œuvre de Bélaïd Ait Ali déjà parue en 1964 sous le titre : Les cahiers de Bélaïd ou la Kabylie d'antan sous le titre (Ițțafțtaren yura Bélcid at Acli af Leqbayel n zzman n zik). 
(certainement par manque de maturité). Le revoilà libre. Il passe son temps entre Azrou, Alger chez ses oncles maternels et en France. A 20 ans, il fut appelé au service militaire. Il gravit les grades jusqu'à sergent chef. Démobilisé, il recommence ses errements en particulier en France où il exerça quelques métiers dans des usines. Vers 1934, il regagne son village pour se remarier avec une fille du village cette fois-ci avec son consentement. Il eut très vite un garçon, Ramdane, mais très vite son bonheur va se briser sur des malentendus survenus entre sa mère et sa femme. Bientôt sa femme quitte le domicile conjugal et rejoint sa mère à Alger emmenant avec elle ce qu'il a de précieux au monde, son fils. A partir de ce jour Bélaïd se trouve désemparé, trahi. Témoin ce poème écrit sur sa femme :

Deg Uzru n at Mangellet

Ayufiy taffat

Yeğょel Rebbi tjerh-iyi.

Faṭima d aclim tečča-t

Hafi ițtetekket

Ttagujilt tyaḍ-iyi.

Ur tnuda d ul-iw tuffa-t

Slascaq $n$ tektabt

Yeččur, yurğa i wimi

Xulfay cwar xtaray-t nețtat

Kra bb ${ }^{w} e h b i b$ neğğa-t

Nniya-s: kem barka-yi!

Annay a Lmalaykkat,

Yak tura yețfat,

Ma skedbay nnayemt-iyi !

Yibb $^{w}$ ass adfel la yekkat

$\mathrm{D}$ aqcic-iw teğğa-t

D idduh yețru : texdec-iyi

Yețyidi win ya yecgen

A kra d-issellen

Ad yeğzel yeshel l'Amour

D lehlek $n$ daymen

Ddwa ulac c'est pour toujours

D ul-im ara kem-ixelșen

A tin ixeddsen

S Rebbi ar d ad d-yas ton tour.
A Azrou des Ait Manguellet

Là où j'ai trouvé la lumière

Dieu a permit qu'elle me blessât

Fatima mangeait du son

Pieds nus elle marchait

J'ai eu pitié de l'orpheline

Sans chercher, elle a trouvé mon coeur

Avec l'amour des livres

Remplit il attendait pour qui

N'écoutant aucun conseil, je l'ai choisie

J'ai abandonné mes amis

Je lui ai dit : toi tu me suffis

Oh! Par tous les Anges

Maintenant que c'est du passé

Si j'ai menti, tuez-moi

Un jour alors qu'il neigeait

Elle a abandonné mon enfant

Dans le berceau il pleurait, elle m'a trahi

De la pitié pour celui qui se trompe

A tous ceux qui écoutent

Qu'ils pensent que l'amour est facile

C'est une maladie de tous les jours

Il n'ya aucun médicament c'est pour toujours

C'est ton cœur qui paiera

A celle qui trahit

Par Dieu, ton tour arrivera un jour 
Bélaïd reprend le chemin de l'exil, il repart en France et sombre dans l'alcool. Bientôt la guerre le rappelle, c'est la mobilisation générale. Il se retrouve envoyé avec son régiment dans le Sud tunisien. La boisson lui fait perdre ses grades et devient simple soldat chargé de l'intendance. En Europe, la guerre fait rage et son régiment est appelé en France occupée. A la veille de son départ, il profite d'une permission pour prendre ses jambes à son cou, il rejoint son village, on ne sait par quel moyen.

C'est à partir de ce moment que sa vie va changer entièrement. Traqué par les gendarmes, il trouve souvent refuge chez ses sœurs mariées dans un village voisin. Il commence aussi à lier connaissance avec un Père Blanc (le Père Degezelle) qui venait lors de ses tournées faire causette avec sa mère. Il faut rappeler que celle-ci était l'une des premières femmes institutrice de la Kabylie. Elle a fréquenté l'école de jeunes filles de Taddart Ufella près de Fort National (Larba at Yiraten). Elle a été élève de cette école dans la même période que Fadma At Mansour Amrouche (la mère de Taos et de Jean Amrouche). D'ailleurs celle-ci l'a citée plusieurs fois dans son livre "L’histoire de ma vie », pages 32 et 37. Elle était son aînée de six ans.

Par la conjonction du besoin des Pères Blancs qui cherchaient à recueillir des textes sur la société kabyle pour alimenter le Fichier de Documentation Berbère (FDB) ${ }^{2}$ et la situation sociale de Bélaïd, déserteur, recherché, abandonné par les siens, débouche à partir de mars I946, en particulier, par l'écriture du premier cahier avec le conte «bu yedmimen » (l'aubépine).

Quand on regarde de plus près ce premier cahier on relève les difficultés de Bélaïd en ce qui concerne la réalisation de cet ouvrage. L'alphabet utilisé est presque similaire à ceux de Boulifa et de Ben Sedira. La semi-consonne «y» est notée « ie ou ia », le «c» est noté en français «ch » le «w » est noté « oua » et beaucoup d'autres phonèmes sont notés en français. Mais dès le deuxième cahier, il introduit d'autres signes pour éclairer le lecteur et lui faciliter la compréhension de ses textes. C'est ainsi qu'il commence à introduire le « $\mathrm{y}$ » et le « w » ainsi qu'à différencier

2 Le FdB est un fichier créé par Les Pères Blancs sous la direction du Père Dallet et dans les fascicules sont édités à Fort National, et recueille des écrits très larges sur la culture et les coutumes sur la Kabylie. 
les phonèmes emphatiques comme le « $\mathrm{z}$ » et le « $\mathrm{z}$ » le « $\mathrm{d}$ » avec son emphatique et sa spirante.

Au fur et à mesure qu'il avance dans l'écriture de ses cahiers il innove et invente un alphabet qui lui est presque propre. Car en 1945 il n'existe aucun alphabet normalisé. Les Pères Blancs eux-mêmes étaient à la recherche de signes pouvant répondre à leur problème. Ce n'est qu'à partir de 1946 que certains signes ont été définitivement adoptés. Comme par exemple le signe « $\varepsilon$ ».

L’ouvre de Bélaïd a donc commencé par un simple conte "Bu yedmimen » (l'aubépine) puis il se laisse coller à la tradition et à sa langue. Contrairement à un Boulifa ${ }^{3}$ qui est soumis à des contraintes professionnelles et scientifiques traduisant des concepts modernes qu'il veut rendre dans sa langue, Bélaïd lui est un conteur, il s'est collé à la tradition et à sa langue. Son expression est tirée du quotidien avec un vocabulaire du peuple sans recherche excessive. Il suffit de lire « Jeddi » (grand père) ${ }^{4}$ pour s'en rendre compte.

D'après une confession de sa femme faite à des membres de sa famille, bien avant sa déchéance, Bélaid consignait déjà ses impressions, en français, sur des bouts de papiers. Car il est curieux de tout ce qu'il voyait et entendait autour de lui. Mais il n'a jamais manifesté une volonté d'écrire en kabyle. Comment peut-il le faire ? Pour lui le kabyle ne s'écrit pas.

Il n'a aucune notion sur l'écriture du kabyle. Mais le Père Degezelle, son seul interlocuteur, ne le lâche pas, il l'encourage et le pousse à écrire un conte. Bélaïd lui répondit :

Il ne m'est doux d'exprimer mes sentiments et j'allais dire, d'une façon sérieuse... que dans cette langue que je vous disais tout à l'heure, encore, avoir parlé dès mes premiers balbutiements du berceau. Je suis kabyle bien sûr... mais mon Père, cela n’empêche que c'est en français que je pense, que mes idées mêmes, se conçoivent, naissent ${ }^{5}$.

3 Si Amar ou Said est né en 1865 à Adeni commune d'irdjen, grande Kabylie. I $\mu$ l est l'auteur de plusieurs ouvrages : Une première année de langue kabyle jourdan 1887, Recueil de poèsies kabyles jourdan 1904 réédité par Yacine Tassadit Editions Awal 1990, Méthode de langue kabyle (cours de 2ème année Jourdan 1913, Le djurdjura à travers l'histoire (deuis l'antiquité jusqu'à 1830). 4 Jeddi voir Les cahiers de Bélaid at Ali, regards sur une oeuvre pionière Tira éditions, 2013, p. 50. 5 Voir Bélaïd Aït Ali. Errance ou génie littéraire, Dar Khattab éditions par Ibrahim M., p. 95. 
En effet jusque dans les années I940, il n'y a pas de production littéraire écrite, juste des collectes de contes, de poésies, de devinettes etc...

Ecoutant ce qu'il écrit encore au Père Degezelle dans une lettre accompagnant le premier cahier :

... Une langue kabyle en « caractères " latins ? Je veux bien, mon Père. Je ne demande pas mieux. Je vous souhaite complète réussite et je vous garantis, enfin, toute le reconnaissance des kabyles, dans les siècles à venir ». Mais dès le deuxième cahier il écrit encore :

Oui mon Père, jamais assez merci, oh ! Pour beaucoup de choses. Et en tout cas - et en premier pour ce que vous voulez mapprendre - et me montrer ... que j'ignorais mon kabyle !

On constate qu'il découvre sa propre langue et il le dit toujours dans une lettre accompagnant le troisième cahier :

Ouf, quel voyage mon Dieu! Quel voyage et cependant quelle douce fatigue ! Il me semble remonter d'un gouffre sans fonds! A tel point qu'à certains moments, j’y ressens ... comme une frayeur. Oui mon Père, je m'effraie - mais quand je vous vois me tenant par la main, je ne moccupe plus que de ... jouir des merveilles qu'on y découvre.

Car - et c'est là l'une des merveilles - je vais de découvertes en découverte, bien que ... en descendant.

Et dire que c'est à vous, mon Père, que je devrais ça. Mais nous parlerons plus tard. Et ... si vous nềtes pas fatigué mon Père. Redescendons.

Son œuvre est composée de plusieurs thèmes :

D'abord quatrecontes : Bu Yedmimen, Awayzniw, Acqa issawalen et Tafunast igujilen : (L'aubépine, l'ogre, le grain magique,la vache des orphelins). Bélaïd ne s'est pas contenté de les écrire tels que nos grands parents nous les racontaient. Il les a transformés en une œuvre littéraire les rendant vivants en s'introduisant parmi les personnages du conte, leur donnant des noms et leur faisant réciter des poèmes de sa composition.

Bélaïd a abordé dans ses cahiers plusieurs thèmes que je classe comme suit :

- Les nouvelles :

Tassa, (le foie) texte sur les rapports enfants parents. 
T,teḍber, (le conseil) une leçon de morale qu'un vieux amusnaw donnait à ses enfants.

At zik (les anciens)

Dayen $d$-hakun (ce qu'ils racontent) où l'on retrouve des petites histoires drôles parfois que l'on raconte sur le comportement des individus (hommes ou femmes) tels que af tazzart, qqaren-as kan leqbayel harcen, ou tamexluqt. (On dit que les kabyles sont rusés ou l'inconnue).

Sya d sya (par-ci par-là)

Lyani d lfaqir (le riche et le pauvre)

Azidan d umerzagu (le sucré et l'amer)

- Il ya aussi des textes que je peux appeler «Tiqșidin ». Ce sont des récits moralisants ayant comme personnages des animaux tels sont les cas de :

Lhăg amcic (le chat pellerin hadj)

Tamacabut inissi d wuccen (le hérisson et le chacal)

Ass mi heddren lehwayec (du temps ou les animaux parlaient)

- D'autres textes peuvent être ethnographiques par exemple :

Jeddi (Grand père)

Af tmusni (Sur l'intelligence)

Lexdubga (les démarches matrimoniales) à lui seul tient un cahier complet Père Degezelle

Mara d-mektiy (quand je me remémore)

$D$ amezwaru unebdu (le premier jour de l'été)

- Par ailleurs il a consacré un cahier complet aussi aux femmes du village :

Dehbiya at Qasi (nom de femme kabyle)

Zayna at Eamruc (nom de femme kabyle)

Tamezwarut ou Amnuy $n$ teqqcicin (la première ou la dispute de fillettes)

Jubra n tbilbuzt (pseudonyme donné à ma grand-mère appelé communément Tillilit étant sa voisine, elle lui procurait de l'eau de source pour la boisson et en échange lui écrivait des lettres destinées à son fils engagé dans la marine). 
Beru (la répudation)

Sbițtar (Scènes d'hôpital où les femmes se rendaient pour des soins prodigués dans le dispensaire)

- Un cahier complet où sont notés une trentaine de poèmes, tous de sa composition.

Mais d'autres poèmes figurent dans d'autres cahiers (pour compléter la fin du cahier ou simplement dans la bouche de ses personnages au milieu d'un conte ou d'un récit).

Enfin il ne faut pas oublier son chef d'œuvre Lwali bb ${ }^{w} e d r a r$ (Le saint de la montagne) qui, à lui tout seul, constitue une œuvre littéraire complète.

Il faut signaler que Bélaïd a utilisé des noms d'emprunt pour décrire des personnages réels tels que sa mère par exemple dans Targit (le rêve) ou Beru (la répudiation) ou encore Afenğal n lqabwa (la tasse de café). Ma grand-mère appelée communément «Tillilit » a pris le sobriquet de «Tabilbuzt »

Ces cahiers, qui sont en réalité des feuilles volantes fournies par le Père Blanc Jules Louis Degezelle sont cousues avec une aiguille et du fil noir, constituent la plus importante production du Fichier de documentation Berbère initié et dirigé par Le Père Dallet. Ils sont édités en I964, c'est-à-dire après l'indépendance de l'Algérie. L'œuvre est passée presque inaperçue à cette époque difficile quand il s'agit de s'intéresser au berbère. Il n'y a que quelques initiés qui ont remarqué cette production. Le premier à en parler fut, nous semble t-il, Vincent Monteil en octobre I967 dans la préface du livre «L'bistoire de ma vie » de Faḍma at Manșour Amrouche. Il disait de lui : «Ziy... comment oublier l'étonnant Bélaïd Ait Ali, mort en I950, dont le fichier berbère des Pères Blancs de Fort-National nous garde précieusement la mémoire ? Sous-officier cassé, ivrogne, clochard, déserteur, de volonté aussi pauvre que son intelligence était belle, il écrivait en français comme en kabyle, et les beaux textes qu'il nous a laissés vont à la rencontre de ceux de Fadhma Amrouche ».

Le deuxième qui a remarqué Bélaïd fut Redjala M'barek éminent intellectuel algérien et qui est le premier kabyle à être sorti diplomé " des Langues $\mathrm{O}$ » ancêtre de l'inalco (Institut National des Langues et Civilisations Orientales) dans un article sur la Kabylie et la culture 
kabyle publié dans l'Encyclopédia Universalis en 1970. Il disait de Bélaïd : «D'ailleurs, le conte, qui a déjà subi des dégradations, semble engagé, bien que timidement, dans une voie qui pourrait déboucher sur la naissance d'une prose consciemment élaborée. Le mérite en revient à Bélaïd Aït Ali dont les Pères Blancs ont publié en 1964, Les Cabiers ou la Kabylie d'antan. Dans une prose empruntée au conte, mais retravaillée et soumise à la contrainte qu'impose la volonté de l'écrit littéraire, ce Kabyle de culture française a raconté des histoires qui tiennent à la fois du conte, du roman et de la confession ».

Puis c'est le silence complet, jusqu'aux événements des années 1980 où les langues commencent à se délier. La première émission radiophonique a été diffusée par radio Beur avec la participation de Ould Braham Idir. Ce dernier récidive en publiant plusieurs articles dans sa revue annuelle «Documents et textes berbères » en I982. Mais la découverte de Bélaïd est sans aucun doute due à sa biographie réalisée dans un mémoire de maitrise qui lui est consacré à l'inalco par Iвrahim Mohand. Ce mémoire légèrement retouché a été édité par les éditions Dar Khettab en 20I5. Auparavant c'est-à-dire dès 2009, il faut noter que le même éditeur Rachid Khettab a numérisé lédition de 1964 pour une diffusion plus large.

Je viens de présenter succinctement la vie et l'œuvre de Bélaïd qui est assez connue maintenant. Mais il reste une autre dimension mal méconnue du public, celle d'artiste peintre. Malheureusement son œuvre pour la plupart est perdue tel un dessin de son village Azrou Kollal réalisé vers les années I940 ou une peinture murale, en couleur, sur la devanture d'un magasin d'un marchand de fruits et légumes, d'un plat rempli de fruits (pastèque, raisin, pomme et poire) effacée par le temps et les hommes inconscients à l'époque de l'importance du sujet.

Il nous reste une aquarelle peinte de sa maison et représentant la montagne du Djurdjura et offerte au Père Degezelle son ami et son confident. Cette peinture est conservée par le Centre de Recherche Berbère de l'Inalco. Elle figure en noir et blanc dans la revue «Textes et Documents Berbères " $\mathrm{n}^{\circ} 2$, année 1982 et en première page de la couverture du livre «Ițtafțțaren yura Bélzid at Asli af Leqbayel n zzman n zik » édition Dar Khettab, en 20I5. Cette nouvelle édition est originale puisque l'auteur (M. IвRAHim) est parti des textes du manuscrit en respectant l'évolution de 


\section{Mohand Ibrahim}

l'écriture des cahiers par Bélaïd et en y insérant les différents échanges qui se sont produits avec le Père Blanc Degezelle, seul interlocuteur de Bélaïd Aït Ali.

\section{ICONOGRAPHIE}

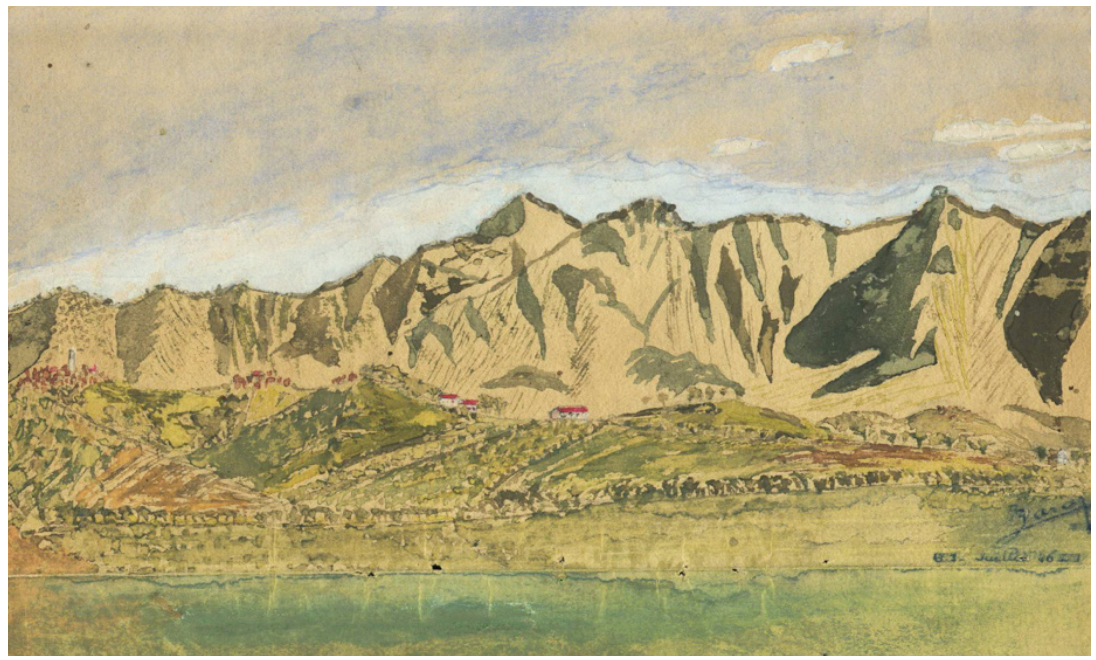

Djurdura, par Belaid

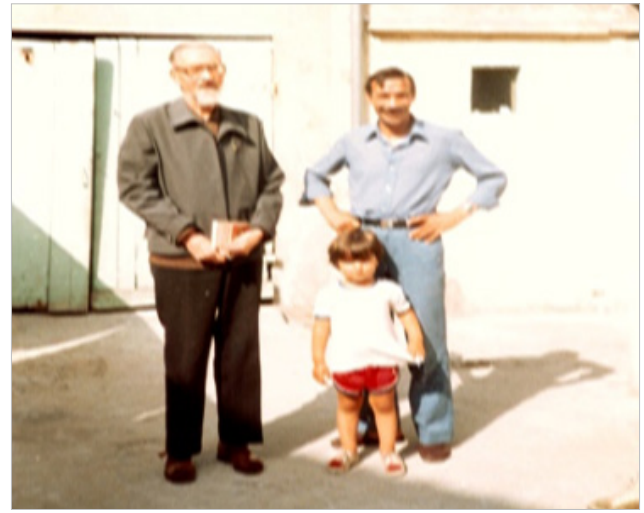

Père Degezelle à gauche dans la cour Acheres avec Mohand Ibrahim, auteur de cet article et un des enfants de celui-ci. 


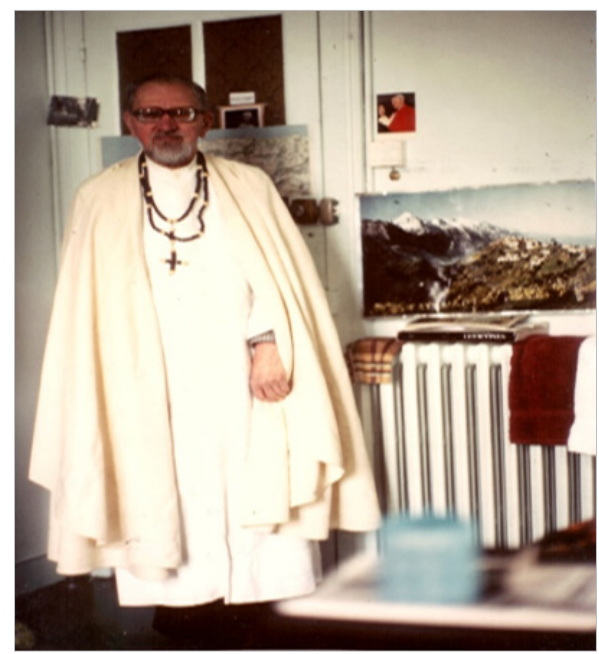

Le Père Degezelle avec l'habit religieux.

Tzem oun ienzzara dachout; istof iefliast iou metoul.

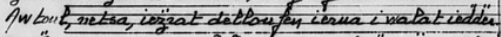

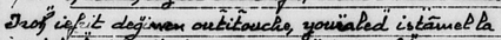
imetchäh ichenfirsio anzoun icietat. Chaçoun

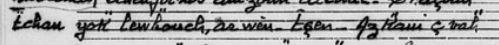

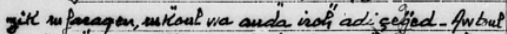

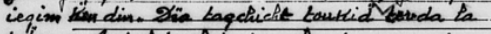
tetsrous. Aolout iwate ts, iscawtes, ienayas:se viga

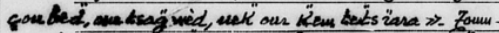

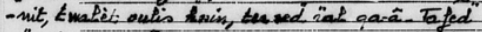

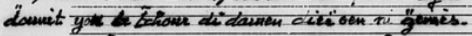

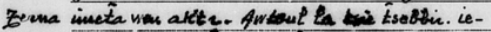

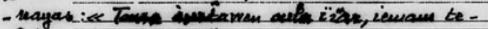

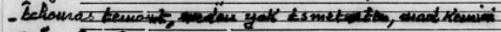

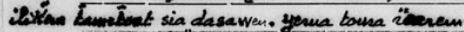

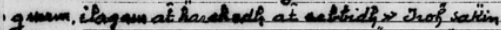

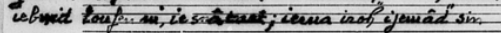

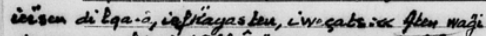

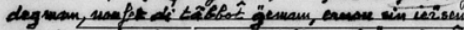

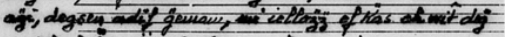

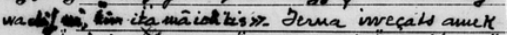

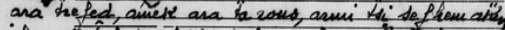

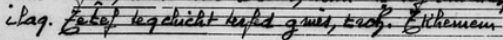

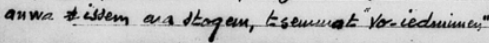

(ahat ini diloul ger isservanen fwedmim of . "Ehol, hat heto, theho... tefai" brimant, bekchem tayedh, elloukn bagi a egicbli - trun dei"k fethis tweit, teçawdhed" än ikwet tad"ent. If wegt ineusi teruter kin rebbi oli teboust ian le- brunt, befwid ain issiektev vol; tEe thaijes.

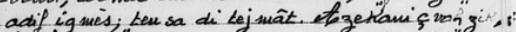
lof jer, nohied iewen mergaz adiezzat, ic fed ra. metorel betes ol Eqaá, iwsenide, Doufeu degchimis. Inah': tametout ticlen.zits, tezien, natehi de iffa "̈nor gavion retbi. Ienchem-Ierva argaz del fahem, iágel sachou ie dh ran iédes- lesteg ïars, is seq seits. Temrayas yor̈ tidets; tehr̈̈anas :

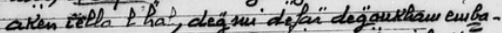

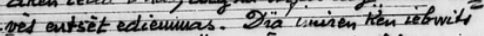

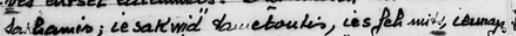

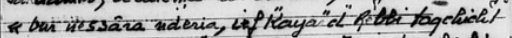

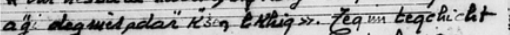

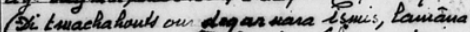

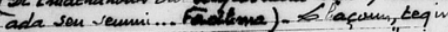

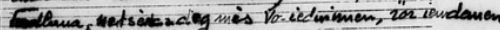

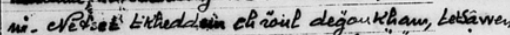

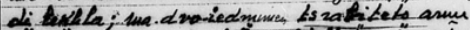

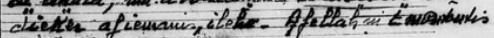
ferhen tèssen diön ken. Oä̈vasmi bekehem

Échantillons de “Les Cahiers” écrits par Belaïd. 
6

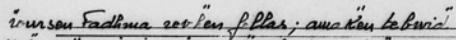

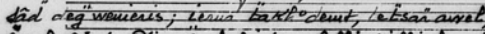

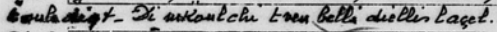

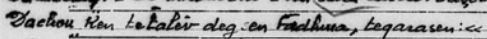

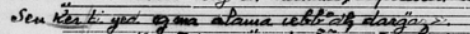

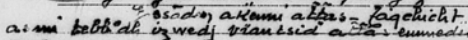

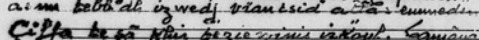

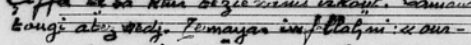

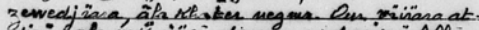

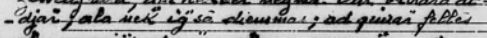

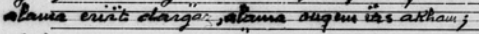

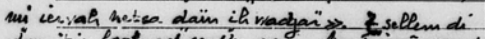

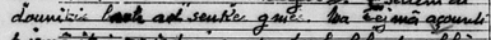

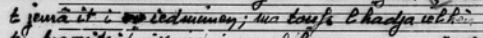

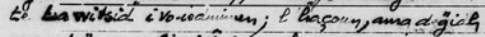

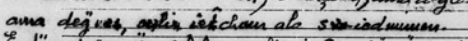

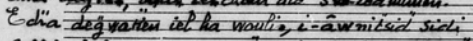

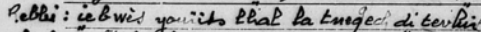

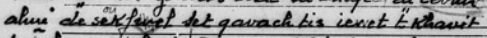

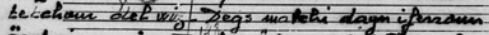

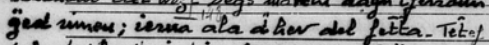

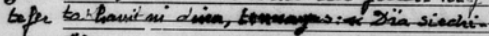

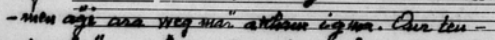

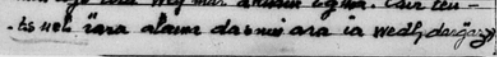

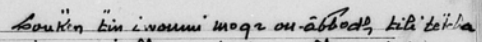
ichinen emi, bla ma ke rethdem, tha dua ta tsev;

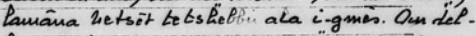

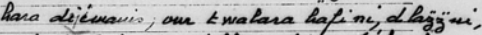

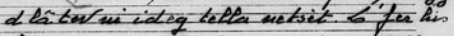

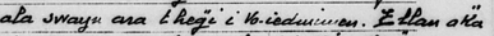

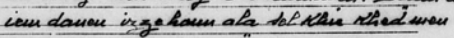

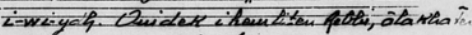
ghen us sere ies hal. Gamana...

"win ithediureus el whin

ie scomialas ols ref mi -

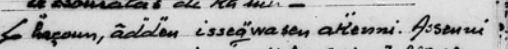

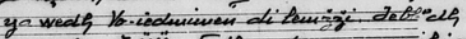

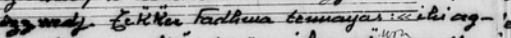

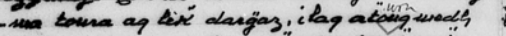

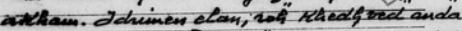

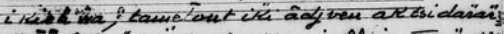

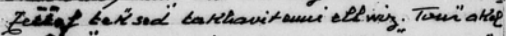

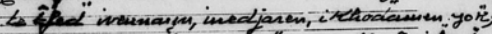

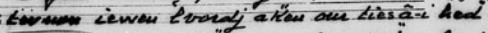

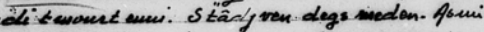

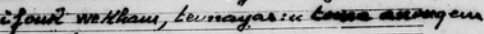
bavier en $\Rightarrow-$

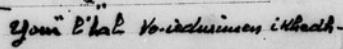

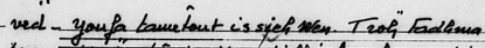

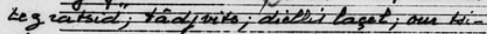

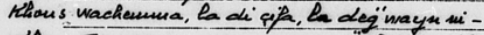

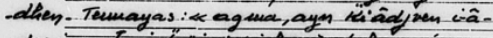

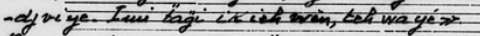

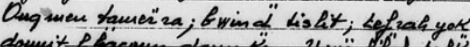

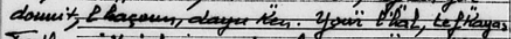

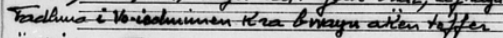

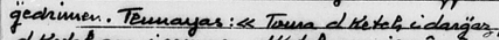
dHetef ara isengen, dHatef asa youm an roniek,

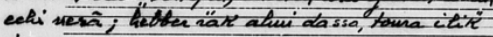

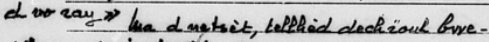

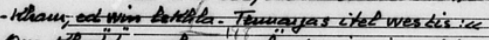

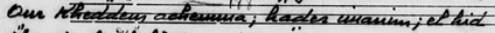

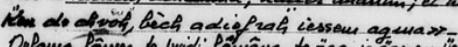

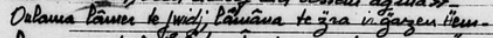

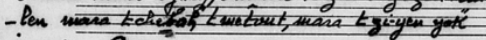

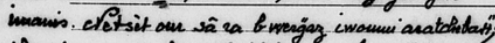

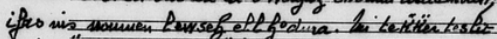

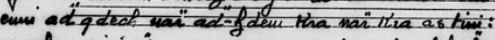

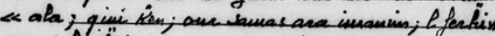

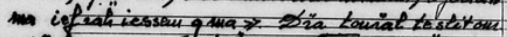

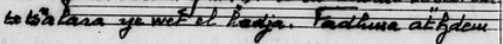

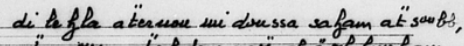
ada goum, at"ef dem yot" of iol bwe tam ha ol Vo-ieduniven, Me rsa choillis, de siada, to follatits, de soug. Iedf-hac outa duetsa delate.

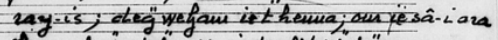
itamu, un roukthadja is teafitidid tgenenes-

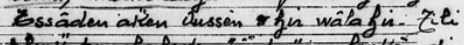

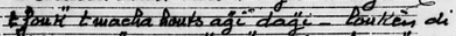
oulich di donimit sta-it" - Kra bwenda ara tili stout adieli char, adiel l heu, adietiva-um

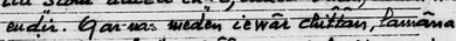
Eg \pm iel vit siant: Chit̂an ma ief zeit-ruadem adierwat filtis, wama stout... (Ienua, dac tow

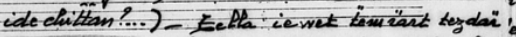

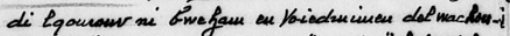

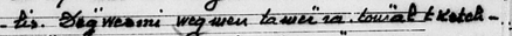

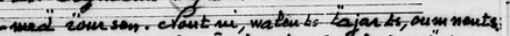
yerna mars teq ine pichoucha (Téstit) Wat des

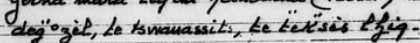

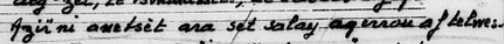

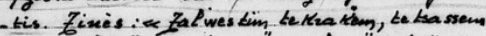

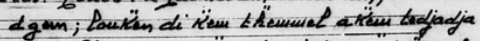

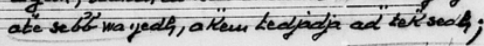


BIBLIOGRAPHIE SUR BÉLAÏD AÏT ALI

Les cabiers de Bélaïd ou la Kabylie d'antan, édition du Fichier de Documentation Berbère 1964.

Histoire de ma vie de Fadhma at Mansour Amrouche, François Maspéro 1982 (préface de Vincent Monteil).

Etudes et Documents berbères n² 1987 dirigé par Ouahmi Ould-Braham.

Redjala, Mbarek. Article sur « la Kabylie », Encyclopédia Universalis vol. 10.

Titouche, R. Les cabiers de Bélaid. Du conte à la nouvelle. Mémoire de Magistère.

KaCI OuALI, Kahina. Le renouvellement littéraire en langue kabyle : le cas de Bélaid at Ali.

Iвrahim, Mohand. Vie de Bélaid at Ali, auteur des cabiers de Bélaid ou la Kabylie d'antan? Analyse et notation du manuscrit, mémoire de maitrise Etudes berbères, Centre de recherche berbère, Inalco, Paris, octubre 1997.

AmÉziane, Amar. Tradition et renouvellement dans la littérature kabyle, thèse de doctorat, Inalco, Paris 2008.

Dallet, J., L. Degezelles (édition). Les cahiers de Bélaid at Ali ou la Kbylie d'Antan, Boudouaou, Dar Khettab, 2009.

Ittaft,taren $n$ Beleid yura af leqbayel $n$ zzman $n$ zik. Réscriture des manuscrits des cahiers de Bélaid avec la notation moderne avec des textes inédits par Ibrahim Mohand, éditions Dar Khattab, 2015.

Ommes et Femmes de Kabylie, sous la direction de Salem Chaker, Ina-yas / Edisud, 2001.

Les cahiers de Bélaid at Ali. Regards sur une oeuvre pionnière, ouvrage dirigé par Dr Amar méziane, Tira éditions, octubre 2013.

Un article ("Bélaid at Ali ; un précurseur ») a été consacré à Bélaid at Ali par Boussad Berrichi dans le journal La nouvelle République n 392 des 20/05/1999 et no 394 du 23/05/1999. 
